\title{
Cell Di-Electrorotation: Studying of Rotation to Characterize Biological Cells and the Connections in Engineering to the Next Generation Standards
}

\author{
Mr. Shawn H Maison, Central Michigan University and Bangor Township Schools
}

Mr. Maison is a high school STEM teacher in Biology, Biotechnology and Anatomy. He also teaches as a Geology, Biology, and Environmental instructor at the collegiate level. He has always been passionate about the natural sciences. Mr. Maison believes that understanding ourselves and the world within and around us is what will drive us as a society. It is also what motivates him as a teacher. He finds true value in science, technology, engineering, and math disciplines and believes those disciplines are the tools our youth desperately need to become successful leaders in the world today. Mr. Maison enjoys helping students realize their biological passion and niche in science. His experiences fuel his desire to continue to learn and teach.

\section{Mr. Adam J. P. Bauer, CMU}

Mr. Bauer is currently in the graduate Chemistry program at Central Michigan University and is doing research with Dr. Bingbing Li. His undergraduate degree from Alma College was in Biochemistry.

\section{Steven Shapardanis, Central Michigan University \\ Thomas Stuart White \\ Mr. Ze Zhang \\ Prof. Bingbing Li, Department of Chemistry, Science of Advanced Materials Doctoral Program, Central Michi- gan University \\ Dr. Qin Hu, Central Michigan University}

Dr. Qin Hu received her B.S. and M.S. degrees in Electrical Engineering from University of Electronic Science and Technology of China in Chengdu, China, and received her Ph.D. degree in Electrical Engineering from Old Dominion University in Norfolk, in 2004. She was a post-doctoral research fellow at Old Dominion University from 2004 to 2007. In 2007, she joined Central Michigan University as assistant professor of Electrical Engineering. She teaches in the area of microelectronic circuits, microprocessor, probability, statistics and random process in engineering. Her main research interests have been in the area of numerical bioelectromagnetics, semiconductor devices modeling \& simulation, electrical properties of materials, therapeutic applications of electromagnetic fields, and software engineering and development. She has authored/co-authored 26 journal papers and several conference papers published in prestigious, international, peer-reviewed journals. She is a senior member of the Institute of Electrical and Electronics Engineers (IEEE), a member of the Biophysical Society (BPS) and a member of the Society of Woman Engineers (SWE).

\section{Dr. Tolga Kaya, Central Michigan University}

Dr. Tolga Kaya currently holds a joint assistant professor position in the School of Engineering and Technology and the Science of Advanced Materials program at Central Michigan University (CMU). Prior to joining CMU, From 2007 to 2010, Dr. Kaya was a post-doctorate associate at Yale University. From 1999 to 2007, he was a research and teaching assistant at Istanbul Technical University. In 2007 he became a consultant at Brightwell Corp. He was a senior VLSI analog design engineer and project coordinator at Microelectronics R\&D Company from 2000 to 2006, and a visiting assistant in research at Yale University from 2004 to 2005. Dr. Kaya received B.S., M.S. and Ph.D. degrees in Electronics Engineering from Istanbul Technical University in Istanbul, Turkey. His research interests in electrical engineering and applied sciences are analog VLSI circuit design, MEMS sensors and energy harvesting systems. His research is also involved in biomedical engineering where bacterial hydrodynamics are studied under various shear flow regimes to enlighten the bacterial infections in catheterized patients. He is also working in engineering education research. 


\title{
Cell Di-Electrorotation: Studying of Rotation to Characterize Biological Cells and the Connections in Engineering to the Next Generation Standards
}

\begin{abstract}
Biological engineering is a field that most secondary educator will never gain research experience in. With new engineering standards playing a predominant role in the new "Next Generation Science Standards" teachers and students will be struggling for meaningful lesson plans that teach engineering standards. Students will need leaders (teachers) that have an engineering knowledge base to properly learn true engineering practice. Teachers will need real engineering experiences to be proficient enough to help students learn true engineering concepts and standards. That is what the RET (Research Experiences for Teachers) has provided.
\end{abstract}

By creating a di-electrophoresis chamber, cell frequency can be observed. This would allow the identification of healthy cells from diseased cells. Theoretically, one method of identifying cells is by the frequency of their rotation within a di-electrical field. By layering one electric field on top of another in a sealed chamber cells can be trapped within a di-electric field in solution. The RET (NSF project) has given each teacher the tools needed to aide students in the new engineering standards. The Di-electrophoresis project required collaboration between three departments on campus. The biology, chemistry, and engineering departments all collaborated on the di-electrophoresis project. Each department played a critical role in combining resources to fabricate a device that could potential trap cells in an electrical chip. This collaboration is what allowed the engineering and technology end to create the di-electrophoresis device. The parameters were set by the cell size. The cells were removed from sub-culture using biochemistry laboratories and staff. Resources in the engineering and technology department were used to fabricate the device. The correct electrical field was designed by an electrical engineer. The resources and collaboration between disciplines is the core of what engineering is.

In this experience teachers learned to use their strengths, but also know when to rely on others with more experience in different disciplines. As a result of this project we have gained an understanding that failure is a teaching point and that each prototype created is a success no matter what the outcome is. Showing students that engineering is fluid and always changing, improving, and evolving. Redesigning current labs to have outcomes that can vary from student to student is one way we have already been able to adjust current curriculum to meet the new standards in engineering. Many sciences have designated designed outcomes in their labs, and expected results in their directed lessons or projects. Allowing students to design their own labs instead of us giving rigid outlines in experiments or projects is becoming a paradigm shift already in how our labs are being taught at both the secondary and collegiate settings. Our students are more engaged in collaborating and group work as a result of this RET project. We 
will provide detailed project description and resulting classroom activities that were developed as a result of the RET project.

\section{Introduction}

Osteoblasts of a mammalian organism (mouse) are used in this experiment. The cell line of osteoblasts are roughly 15-30 $\mu \mathrm{m}$. in diameter and, if not scaffolding, are generally spherical in shape. This allows for a symmetrical shape to observe rotation accurately in an E-Field in solution. These cells are cultured, and then subcultured, with an optimal 1-2 day incubation between cultures. This insures scaffolding does not take place (If scaffolding begins cells are no longer spherical/symmetrical for measuring rotation). Cell population must also be kept to a minimum to insure cells do not clump together and form globular structures that can't be measured or trapped in an E-field. The osteoblasts are kept alive in a cell nutrient medium which contains penicillin to prevent unwanted bacterial growth, and essential minerals and nutrients (FBS/Fetal Bovine Solution). This fluid is conductive so a barrier between electrodes must be in place. A spun layer of photo-resist serves this purpose.

The di-electrorotation chamber fabricated is two sets of electrotrode circuits that overlap to form a grid to trap cells. The electrodes are kept separate from each other to create the field by having a taped barrier in place to create a well between the two electrodes. The cell solution is then pumped into the di-electrically charged chamber. The cells are theoretically trapped within the di-electrically charged grid. The cells theoretically rotate at a frequency based on their cell membrane's structure and charge.

As new engineering science standards enter high school classrooms around the country teachers will need real engineering experiences. This National Science Foundation Project and Science Math Teacher Center at Central Michigan University project has given high school teachers an applied scientific and engineering application. Biological engineering is an exploding field at this time and giving students a real idea of what is entailed in a real engineering research project will prove invaluable in their engineering and science education. This experience has shown how collaboration, non-defined outcomes, and analytical thinking are the essential backbone of the engineering field. 


\section{Subculturing Osteoblasts}

Osteoblasts are bone cells that are used to remodel bone tissue. These are the cells that first form a bony callus to repair damaged compact bone. When they are repairing bone they scaffold out to create layers of bone much like fiberglass. It is this scaffolding that gives bone its structure and function. When the cells are scaffolding they appear dendritic. See figure 1 to observe this scaffolding ${ }^{1}$. Prior to scaffolding the osteoblasts are in a spherical shape. It is this spherical symmetrical shape that is needed to obtain a uniform diameter for observing cell rotation. See figure 2 to observe cells non-scaffolded in trypsin ${ }^{1}$.

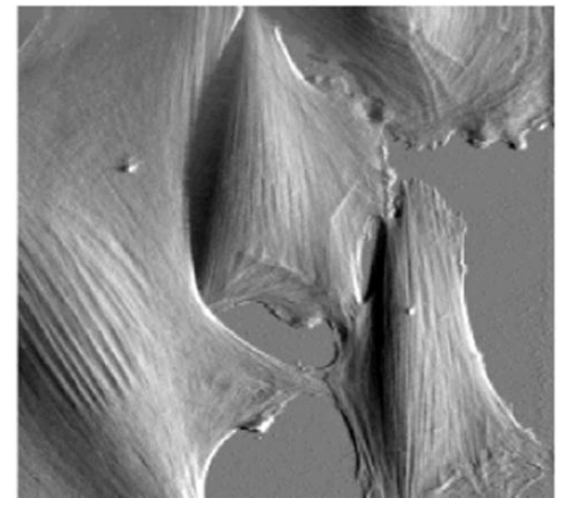

Figure 1: Osteoblasts fully scaffolding ${ }^{1}$.

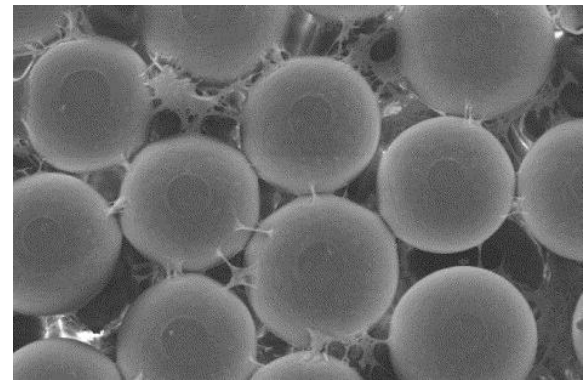

Figure 2: Osteoblasts in their spherical shape just before scaffolding begins ${ }^{1}$.

When osteoblasts are subcultured they need to be removed from the glass petri dish where they adhere to. In this stage they are scaffolding and are not spherical in structure. Trypsin is used to remove the cells from the dish. Trypsin accomplishes this by sending cells into a survival state. If trypsin is not used the osteoblasts will remain on the substrate (petri dish), and not be held in solution. The cells will bundle up and the dendrite like structures will retract inward to the cell forming a spherical shape. This is the point at which the cells need to be taken to run test trials and when cell rotation can be measured.

The cells will not stay in this spherical shape for long. They are not able to stay in the trypsin, and must be put in the new cell medium solution to keep them alive. The solution consists of 2.2 grams of $\mathrm{Na}_{2} \mathrm{HCO}_{3}, 1 \%$ Penicillin, and $10 \% \mathrm{FBS}$ (Fetal Bovine Solution) in aqueous solution per $100 \mathrm{ml}$. The difficulty is that once they are placed in the cell medium solution they will start to 
scaffold and dendrite out again. This will occur within one hour of sub-culturing (usually between 30-60 minutes). The experiment must be run within that time frame or the cells will not be symmetrical, and will therefore not show accurate rotation. The concentration that we will use is 100,000 cells per ml. of cell medium. A hemocytometer is used to obtain a cell count and obtain the desired solution as seen in figure $3^{2}$.

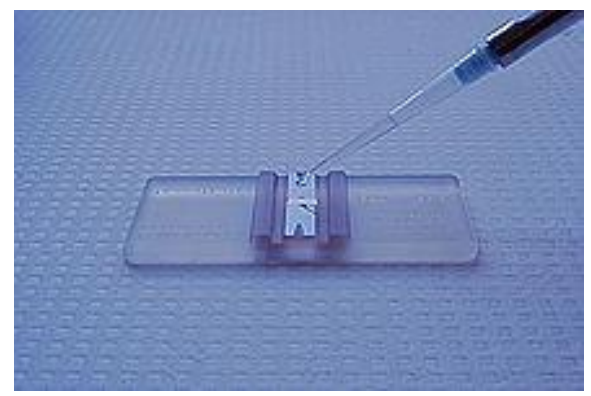

Figure 3: Dead cells stained with trypan blue will not be counted and an accurate dilution can be created with an accurate living cell population count ${ }^{2}$.

\section{Etching Copper Electrodes to create electrical grid}

The pattern for the device is two intertwined circuits that run parallel to each other. The pattern is etched onto copper coated slides. In order to do this masking and photoresist must be used in a developing process. The masking pattern is show below in figure 4. First, a maskaliner must be used to put a pattern on a copper coated glass slide to positively expose the desired template. Plan copper coated glass slide is shown below in figure 5. Once this is accomplished the copper can be developed. The next step is to put photoresist on the desired pattern to only leave the circuit pattern behind when developed. This must be done in a dark room environment.
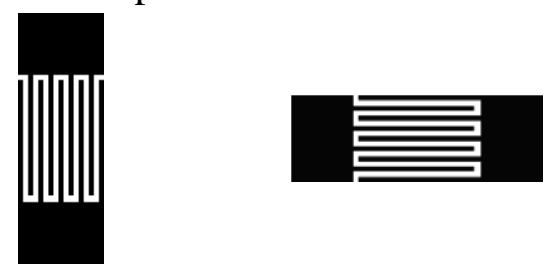

Figure 4: Masking Pattern developed on copper coated glass slides.

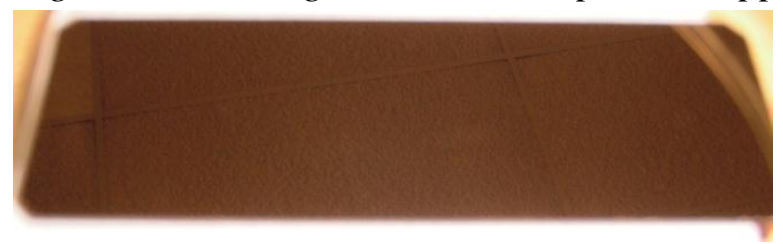

Figure 5: Copper coated glass slide.

Once the desired circuit pattern is developed on the copper coated glass slide the pattern can be etched out. $\mathrm{NA}_{2} \mathrm{~S}_{2} \mathrm{O}_{8}$ is used to etch out the portion of the slide that is not developed. It will be dissolved away in $\mathrm{NA}_{2} \mathrm{~S}_{2} \mathrm{O}_{8}$ removing the unwanted copper that is not masked. The masked undeveloped area will remain. The slides are then cleaned leaving behind only copper in the 
pattern of the circuits desired for the device. The fully etched pattern can be seen below in figure 6.

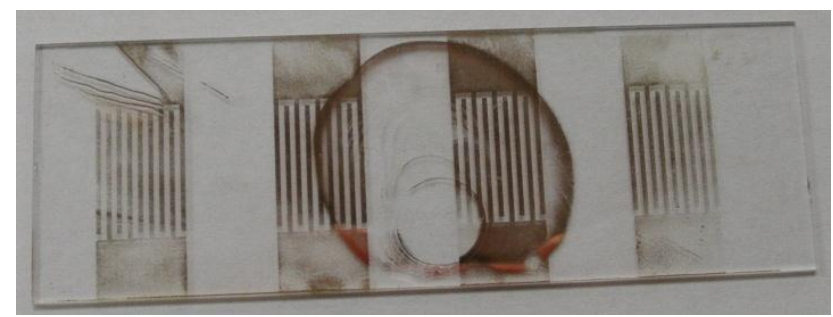

Figure 6: Circuit pattern etched from copper coated slide.

\section{Coating Copper Electrodes with Photoresist}

The cell medium is homeostatic to the osteoblasts and is needed as a medium to keep them alive. It is also highly conductive. It is for this reason that an insulating barrier must be put in place over the copper to prevent the device from shorting out. Because photoresist is hydrophobic, and is not conductive, it will prevent the device from shorting out. Before building the device the circuits must be spun with the photoresist. Then when the medium with osteoblasts in it is put through the device it will not create a short. The layer of photoresist is thin, and it does not need to be kept in a dark room environment. It can be exposed without concern for altering the device or altering the translucence enough to not be able to view the cells under a microscope. These coated devices can be seen below in figure 7 .

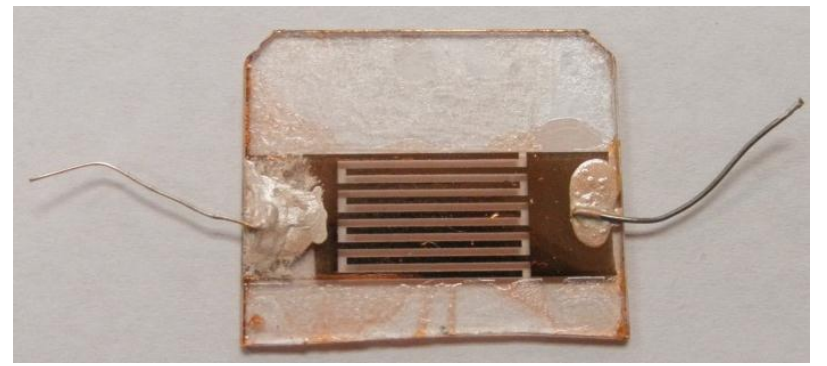

Figure 7: Photoresist coated devises with electrodes (2 will be needed to create one device).

\section{Trapping Osteoblasts in the di-electrical grid:}

When the circuits are put perpendicular to each other they create a grid pattern. The edges of the devices are taped together on two sides with double sided sticky tape. The middle is left open. Two micropipette tips are flattened on each end in a vice. This allows them to pass between the slides from the open ends. They will serve as the ports for the medium and cells. The open space is left open on each end to allow capillarity within the chamber. Figure 8 below shows the completed device will 2 perpendicular devises. 


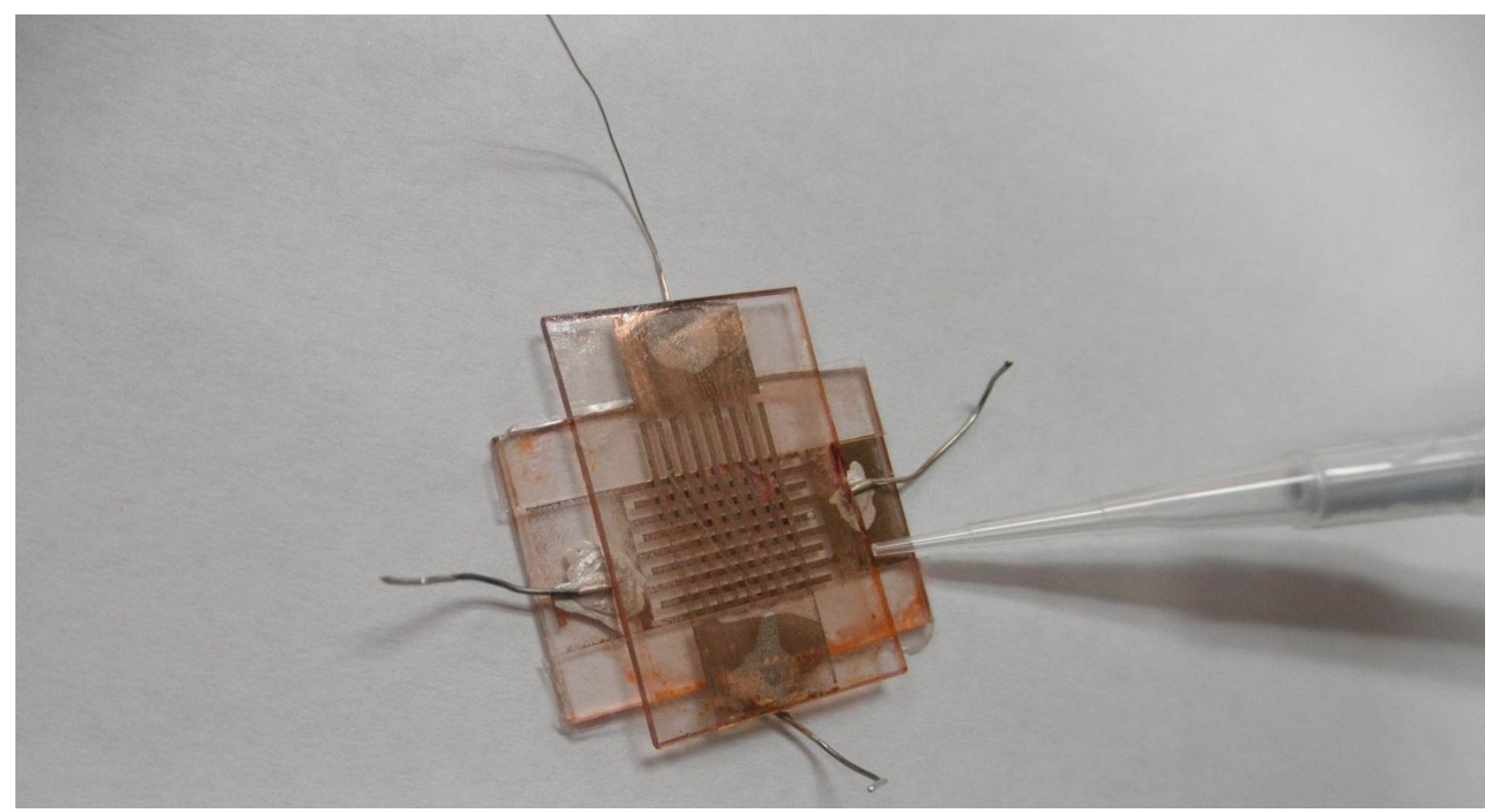

Figure 8: Completed devise with 2 perpendicular gridded circuits creating the desired field.

When cells are pipetted into the chamber in medium, as seen above in figure 8 , they theoretically will be trapped by the field that is created from the overlapping charged circuits. The grid can be seen in figure 9 below under a microscope. The cells obtain a slight negative charge and will hold inside the gridded pattern within an E-field. The E-field is created by conducting a $90^{\circ}$ pulse from each of the four pads on the device. This E-field is illustrated in figure 10 below based on Chengjun Huang's research, and also cells trapped in the E-field can be seen in figure $11^{3}$. This precise $90^{\circ}$ pulse is what allows the trapping of cells to be possible. If rotation occurs it can be observed under a microscope. This is called electrorotation. The middle of each grid should have no charge, but the E-field surrounding the cell(s) should hold them within the charged electric fields.

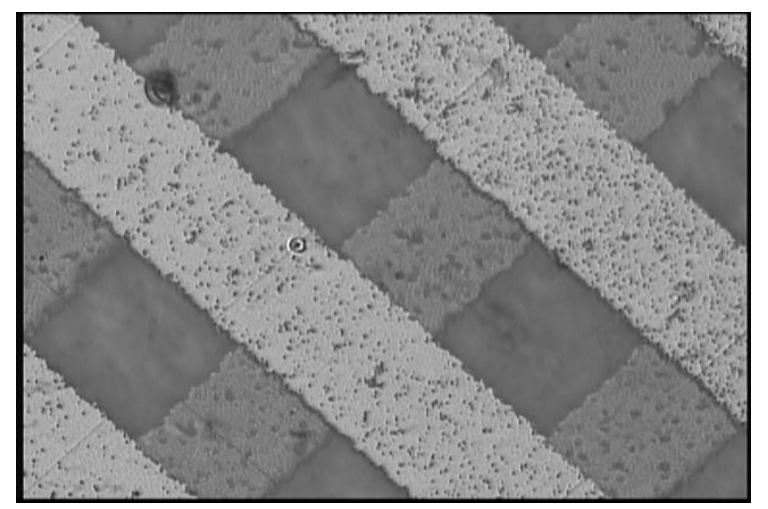

Figure 9: Empty devise with an empty grid created by the 2 perpendicular devises under a microscope. 


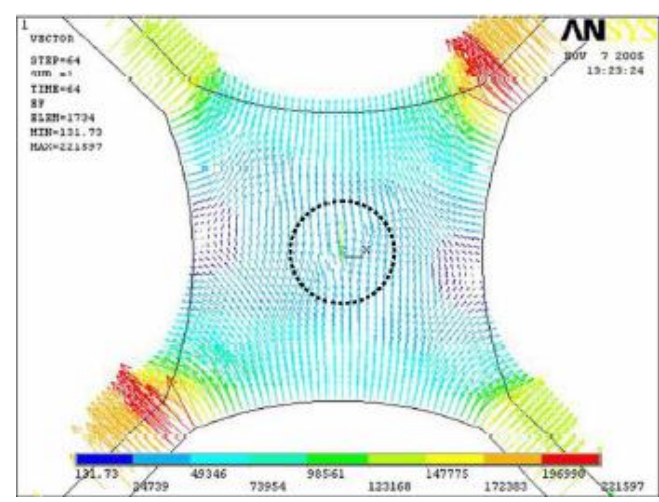

Figure 10: E-field created by a $90^{\circ}$ Pulse ${ }^{3}$.
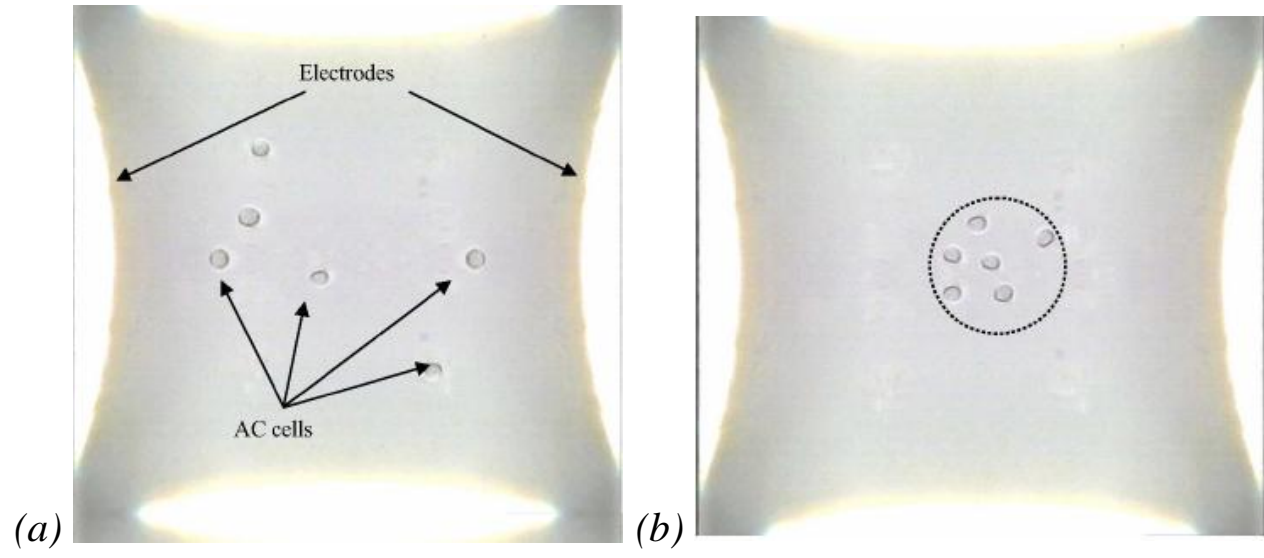

Figure 11: Cells before E-field is created (a) and cells after E-field is turned (b) on pushing cells into the center of the grid $^{3}$.

\section{Measuring Rotation within electrical grid:}

The frequency, hertz, can be measured and compared to other cells. It is the intent to eventually be able to track the rotation of a healthy cell compared to a diseased or possible cancerous cell. The solution that is pumped through the chamber could be blood eventually and be used as a early detection device.

\section{Simulation of Experiment using Microbeads:}

Osteoblasts are delicate in culture, and put tests and experiments on a short time frame. Microbeads will be used to simulate the osteoblast behavior. The microbeads used are tagged fluorescent green. This allows them to be visually tracked. The beads will be mixed in the same cell medium solution that the osteoblasts are cultured in to insure constants in testing. The micro-beads used are $15 \mu \mathrm{m}$ (osteoblasts are 15-30 $\mu \mathrm{m}$. depending on their state... scaffolding or not scaffolding respectively).

\section{RET/NSF/SMTC Project}


Biological engineering is a field that most secondary educator will never gain research experience in. With new engineering standards playing a predominant role in the new "Next Generation Science Standards" teachers and students will be struggling for meaningful lesson plans that teach engineering standards. Students will need leaders (teachers) that have an engineering knowledge base to properly learn true engineering practice. Teachers will need real engineering experiences to be proficient enough to help students learn true engineering concepts and standards.

The NET/NSF/SMTC project at Central Michigan University has given each teacher the tools needed to aide students in the new engineering standards. This project required collaboration between three departments on the Central Michigan University campus. The biology, chemistry, and engineering and technology departments all collaborated on the di-electrophoresis project. Each department played a critical role in combining resources to fabricate a device that could potential trap cells in an electrical chip. This collaboration is what allowed the engineering and technology end to create the di-electrophoresis device. The parameters were set by the cell size. The cells were removed from sub-culture using biochemistry laboratories and staff. Resources in the engineering and technology department were used to fabricate the device. The correct electrical field was designed by an electrical engineer. These resources and collaboration between disciplines is the core of what engineering is.

In this experience teachers learned to use their strengths, but also know when to rely on others with more experience in different disciplines. Understanding that failure is a teaching point and that each prototype created is a success no matter what the outcome is. This will show students that engineering is fluid and always changing, improving, and evolving. Redesigning current labs to have outcomes that can vary from student to student is one way teachers will be able to adjust current curriculum to meet the new standards in engineering. Many sciences have designated designed outcomes in their labs, and expected results in their directed lessons or projects. Allowing students to design their own labs, experiments or projects will be a huge shift in how sciences are being taught in both secondary settings and collegiate settings.

\section{Hemocytometer Cell Counting Lab}

As a participant I was able to develop a lab based on a common tool used to count mammalian cells called a Hemocytometer (seen in figure 9 above). This tool has precise laser etched grids in it that allow the scientist/student the ability to take a basic population or concentration of cells in various grid patterns. With basic calculations one is able to take an accurate cell concentration/population count of any cell population that is in its field. In my research we used it to determine the dilution of cells we would need to subculture mammalian osteoblasts. 
The same concepts and tool can be applied to any eukaryotic cell lab. Similar grids are available to count bacterial cells, but the Hemocytometer has been developed for red blood cell counting. I created a basic lab template that can be used to count a blood sample for red blood cells, or converted to an ecological study counting algae, protozoan, or other pond dwelling single celled or colonizing organisms.

Finding these tools and identifying how to incorporate them into secondary science curriculum will be a main focus in future research opportunities. When students can find real meaning in their curriculum and use practical tools in the classroom it makes their learning experience that more meaningful.

\section{Conclusion:}

Di-electrophoresis is a possible method of trapping cells. The successful trapping of osteoblasts was not accomplished in the experiments ran. A prototypic di-electrophoresis chamber was successfully fabricated with two opposing grids. Osteoblasts were observed within the grid. However, the did not appear to be more or less concentrated within the grid compared to outside the grid. There are several possibilities of error that are present in the current prototype.

Copper etching is not a precise method, and the etched patterns were not perfect. The edges and borders of the circuits showed disconformity under a microscope and could have given an uneven field. The disconformity ran throughout the entire device and raises several concerns as to if a new method or medium needs to be used for copper etching this device.

Alignment is another degree of error in the actual pairing of the two circuits to create the device. The circuits were aligned by manual pairing and no precision tools or instruments were used to align them. A maskaliner should be used in future alignment and pairing of circuits to fabricate these devices.

The DC generator used to create the $90^{\circ}$ pulse was under powered and was not giving accurate output. Calibration must be done to insure the correct current is being uniformly passed through each electrode.

Overall the design of a di-electrophoresis chamber was successful. I am confident that by eliminating the three possible areas of error that are identified a device can be created to trap osteoblasts, and measure cell rotation if it exists. The osteoblasts were highly visible in the grid. In a properly fabricated device with the correct $90^{\circ}$ pulse running osteoblast should be effected by the E-field created. Future testing and fabricating is needed to create a more precise device that will trap cells and measure cell rotation within an E-field. 
The educational experiences in this engineering project will prove to be invaluable for secondary educators. Each teacher in this experience obtained new strategies for developing lessons, labs, and classroom projects. Allowing students to design the actual activity and define the parameters gives them the engineering skillsets needed. There is a need for students to understand that science and engineering do not usually have a defined perfect outcome, and that research is ever evolving and developing, and that this will allow them to think critically and analytically in a collaborative environment. After this experience I see teachers' classrooms changing in ways that will make their teaching more meaningful, analytical, and project based.

\section{Bibliography}

${ }^{1}$ Havelaar, A., \& Brul, S. (2009, May). Science direct. Retrieved from http://dx.doi.org/10.1016/j.bbr.2011.03.031

${ }^{2}$ Caprette, D. (2000, May 11). Experimental biosciences. Retrieved from http://www.ruf.rice.edu/ bioslabs/methods/microscopy/cellcounting.html

${ }^{3}$ Chengjun Huang. (2006). Negative dielectrophoretic force assisted determination differences between autotrophic and heterotrophic algal cells using electrorotation chip. Conference on Nano/Micro Engineered and Molecular Systems, January 18-21, 